PROCEEDINGS OF THE

AMERICAN MATHEMATICAL SOCIETY

Volume 138, Number 5, May 2010, Pages 1853-1859

S 0002-9939(10)10192-0

Article electronically published on January 14, 2010

\title{
DIAMOND, GCH AND WEAK SQUARE
}

\author{
MARTIN ZEMAN
}

(Communicated by Julia Knight)

\begin{abstract}
Shelah proved recently that if $\kappa>\omega$ and $S \subseteq \kappa^{+}$is a stationary set of ordinals of cofinality different from $\operatorname{cf}(\kappa)$, then $2^{\kappa}=\kappa^{+}$implies $\diamond_{\kappa^{+}}(S)$. We show that for singular $\kappa$, an elaboration on his argument allows us to derive $\diamond_{\kappa^{+}}(T)$ from $2^{\kappa}=\kappa^{+}+\square_{\kappa}^{*}$ where $T=\left\{\delta<\kappa^{+} \mid \operatorname{cf}(\delta)=\operatorname{cf}(\kappa)\right\}$. This gives a strong restriction on the existence of saturated ideals on $\kappa^{+}$.
\end{abstract}

\section{INTRODUCTION}

It is a well-known fact that $\nabla_{\lambda}$ implies $2^{<\lambda}=\lambda$. In many situations the converse is also true. Jensen 3 proved that $\mathrm{CH}$ does not imply $\diamond$, so when looking for the converse one has to focus on $\lambda>\omega_{1}$. Let

$$
S_{\mu}^{\lambda}=\{\delta<\lambda \mid \operatorname{cf}(\delta)=\mu\}
$$

and

$$
T_{\kappa}=S_{\mathrm{cf}(\kappa)}^{\kappa^{+}} .
$$

Gregory observed that GCH below $\omega_{2}$ implies $\nabla_{\omega_{2}}\left(S_{\omega}^{\omega_{2}}\right)$. A sequence of improvements on his result, mainly by Gregory [5], Jensen (unpublished) and Shelah [9], resulted in the following theorem, whose proof can be found in [2].

Theorem 0.1 (Gregory, Jensen, Shelah). If $2^{<\kappa}=\kappa$ and $2^{\kappa}=\kappa^{+}$, then $\nabla_{\kappa^{+}}(S)$ holds whenever $S \subseteq \kappa^{+}$is a stationary set of ordinals of cofinality different from $\operatorname{cf}(\kappa)$. If $\kappa$ is singular and additionally $\square_{\kappa}$ holds, then $\nabla_{\kappa^{+}}\left(T_{\kappa}\right)$.

Shelah also proved that for regular $\kappa$, the condition $2^{\kappa}=\kappa^{+}+\square_{\kappa}$ is not sufficient to guarantee $\nabla_{\kappa^{+}}\left(T_{\kappa}\right)$, so the absolute ZFC result is possible only for singular $\kappa$.

The question remained whether the localized GCH, i.e. the equality $2^{\kappa}=\kappa^{+}$, alone implies $\nabla_{\kappa^{+}}$. Shelah proved this to be true for sufficiently large $\kappa$, and recently [10] found an argument that proves it for every uncountable cardinal $\kappa$; see Komjáth's paper [7] for a simplified proof and an elaboration on Shelah's argument.

Theorem 0.2 (Shelah). Let $\kappa>\omega$ and $2^{\kappa}=\kappa^{+}$. Then $\nabla_{\kappa^{+}}(S)$ holds for every stationary $S \subseteq \kappa^{+}$that is disjoint from $T_{\kappa}$.

This note combines arguments from the proof of Theorem 0.1 with Shelah's argument for Theorem 0.2 to give a proof of $\nabla_{\kappa^{+}}(S)$ for $S \subseteq T_{\kappa}$.

Received by the editors February 3, 2009.

2010 Mathematics Subject Classification. Primary 03E04, 03E05.

Key words and phrases. Diamond, weak square, generalized continuum hypothesis.

The author was supported in part by NSF grant DMS-0500799.

(C)2010 American Mathematical Society 
Theorem 0.3 (Main Theorem). Assume $\kappa$ is a singular cardinal and $T \subseteq T_{\kappa}$ is stationary with stationarily many reflection points. Then

$$
2^{\kappa}=\kappa^{+}+\square_{\kappa}^{*} \Longrightarrow \diamond_{\kappa^{+}}(T) \text {. }
$$

It was proved by Cummings, Foreman and Magidor [1] that for singular $\kappa$, the principle $\square_{\kappa}^{*}$ is consistent with the requirement that every stationary $T \subseteq T_{\kappa}$ has stationarily many reflection points. Consequently, in their model we have $\diamond_{\kappa^{+}}(S)$ for all stationary $S \subseteq \kappa^{+}$.

Corollary 0.4. Assume $\kappa$ is a singular cardinal. Then

$$
2^{\kappa}=\kappa^{+}+\square_{\kappa}^{*} \Longrightarrow \nabla_{\kappa}\left(T_{\kappa}\right) .
$$

Both the above theorem and its corollary provide a very strong restriction on the existence of saturated ideals on $\kappa^{+}$and provide a close link between the study of such ideals and PCF theory.

Rinot [8] recently extended the result of this paper to the situation where weak square is replaced by a variant of the approachability property and also showed that, relative to the existence of a supercompact cardinal, it is consistent that $\square_{\kappa}^{*}$ fails but $\nabla_{\kappa^{+}}(S)$ holds for every stationary $S \subseteq \kappa^{+}$. Also, by a recent result of Gitik and Rinot 4, the failure of $\diamond_{\aleph_{\omega+1}}(S)$ is consistent with GCH $+\mathrm{AP}_{\aleph_{\omega}}$, again relative to a supercompact cardinal.

\section{The ARgument}

We begin with splitting Shelah's argument into two steps. We first isolate a combinatorial statement that alone implies the existence of a $\diamond_{\lambda}(S)$-sequence in ZFC; we denote this statement by $\bigcirc_{\lambda}(S)$. This statement is implicit in the arguments in Shelah [10] and Komjáth [7]. It turns out that the implication $\bigcirc_{\lambda}(S) \Longrightarrow \diamond_{\lambda}(S)$ is true regardless of whether $\lambda$ is a successor cardinal or not. The second step is a proof that $\bigcirc_{\lambda}(S)$ holds, which relies on the localized GCH if $\lambda=\kappa^{+}$and $S$ concentrates on points of cofinality distinct from $\mathrm{cf}(\kappa)$, giving the original Shelah's result, and on the weak square if $\kappa$ is singular and $S$ concentrates on points of cofinality $\operatorname{cf}(\kappa)$, giving the result in Theorem 0.3. Our approach owes a lot to Komjáth's exposition in 7].

Definition 1.1. Let $\lambda$ be a regular cardinal and $S \subseteq \lambda$. We say that the pair $\left\langle x_{\xi} \mid \xi<\lambda\right\rangle,\left\langle A_{\delta} \mid \delta \in S\right\rangle$ witnesses $\bigcirc_{\lambda}(S)$ iff the following three conditions are met.

(a) $\left\langle x_{\xi} \mid \xi<\lambda\right\rangle$ is an enumeration of $[\lambda]^{<\lambda}$.

(b) $A_{\delta} \subseteq \delta$ and $\operatorname{card}\left(A_{\delta}\right)<\operatorname{card}(\delta)$ whenever $\delta \in S$.

(c) For every $Z \subseteq \lambda$ there is a stationary $S^{\prime} \subseteq S$ such that for every $\delta \in S^{\prime}$ there are unboundedly many $\alpha<\delta$ for which there is $\beta<\delta$ satisfying $\alpha, \beta \in A_{\delta}$ and $Z \cap \alpha=x_{\beta}$.

We say that $\bigcirc_{\lambda}(S)$ holds iff there are $\left\langle x_{\xi}\right\rangle_{\xi}$ and $\left\langle A_{\delta}\right\rangle_{\delta}$ as above.

Notice that $\bigcirc_{\lambda}(S)$ postulates the existence of an enumeration of $[\lambda]^{<\lambda}$ of length $\lambda$, so it imposes some constraints on the behaviour of the exponential function below $\lambda$. In particular, if $\lambda=\kappa^{+}$, then $\bigcirc_{\lambda}(S)$ implies $2^{\kappa}=\kappa^{+}$. Notice also that (b) in the above definition stipulates that the cardinality of $A_{\delta}$ be strictly smaller than that of $\delta$, which together with (c) implies that without loss of generality $S$ can be viewed as a set of singular ordinals. Of course, this has a non-trivial meaning only when $\lambda$ is inaccessible. Finally observe that if there is a pair $\left\langle x_{\xi} \mid \xi<\lambda\right\rangle$, 
$\left\langle A_{\delta} \mid \delta \in S\right\rangle$ witnessing $\bigcirc_{\lambda}(S)$, then for every enumeration $\left\langle x_{\xi}^{\prime} \mid \xi<\lambda\right\rangle$ there is a sequence $\left\langle A_{\delta}^{\prime} \mid \delta \in S\right\rangle$ such that the pair $\left\langle x_{\xi}^{\prime}\right\rangle_{\xi},\left\langle A_{\delta}^{\prime}\right\rangle_{\delta}$ witnesses $\bigcirc_{\lambda}(S)$. To see this, pick any $f: \lambda \rightarrow \lambda$ such that $x_{\beta}=x_{f(\beta)}^{\prime}$ for all $\beta<\lambda$ and let $A_{\delta}^{\prime}=A_{\delta} \cup f\left[A_{\delta}\right]$ for all $\delta \in S$ satisfying $f[\delta] \subseteq \delta$.

Lemma 1.2. Let $\lambda$ be a regular cardinal, $S \subseteq \lambda$ and $\bigcirc_{\lambda}(S)$ hold. Then there is a pair $\left\langle x_{\xi} \mid \xi<\lambda\right\rangle,\left\langle A_{\delta} \mid \delta \in S\right\rangle$ satisfying the following:

(a) $\left\langle x_{\xi} \mid \xi<\lambda\right\rangle$ is an enumeration of $[\lambda \times \lambda]<\lambda$.

(b) $A_{\delta} \subseteq \delta$ and $\operatorname{card}\left(A_{\delta}\right)<\operatorname{card}(\delta)$ whenever $\delta \in S$.

(c) For every $Z \subseteq \lambda \times \lambda$ there is a stationary $S^{\prime} \subseteq S$ such that for every $\delta \in S^{\prime}$ there are unboundedly many $\alpha<\delta$ for which there is $\beta<\delta$ satisfying $\alpha, \beta \in A_{\delta}$ and $Z \cap(\alpha \times \alpha)=x_{\beta} \cap(\alpha \times \alpha)$.

Proof. Pick a pair $\left\langle y_{\xi} \mid \xi<\lambda\right\rangle,\left\langle B_{\delta} \mid \delta \in S\right\rangle$ witnessing $\bigcirc_{\lambda}(S)$. Let $f: \lambda \times \lambda \rightarrow \lambda$ be a bijection and $C_{f}=\{\delta<\lambda \mid f[\delta \times \delta]=\delta\}$.

For each $\delta \in S$ pick $C_{\delta}$ to be a subset of $\lim \left(C_{f}\right) \cap \delta$ of size strictly smaller than $\operatorname{card}(\delta)$ that is cofinal in $\delta$ if such a set exists; let $C_{\delta}=\varnothing$ otherwise. Letting $x_{\beta}=f^{-1}\left[y_{\beta}\right]$ and $A_{\delta}=B_{\delta} \cup C_{\delta}$, we obtain a pair $\left\langle x_{\xi}\right| \xi\langle\lambda\rangle,\left\langle A_{\delta} \mid \delta \in S\right\rangle$ as in the conclusion of the lemma. To see this, it suffices to verify clause (c) in the statement of the lemma.

Given any $Z \subseteq \lambda \times \lambda$, let $S^{\prime} \subseteq S$ be the stationary set obtained by applying $\bigcirc_{\lambda}(S)$ to $f[Z]$. Let $\delta \in S^{\prime} \cap \lim \left(C_{f}\right)$. If $\bar{\alpha}<\delta$, pick $\alpha \in C_{\delta}$ such that $\bar{\alpha} \leq \alpha$. Since $S^{\prime}$ satisfies (c) in Definition 1.1 with $f[Z], y_{\beta}$ and $B_{\delta}$ in place of $Z, x_{\beta}$ and $A_{\delta}$, there are $\alpha^{\prime}, \beta \in B_{\delta}$ such that $\alpha \leq \alpha^{\prime}$ and $f[Z] \cap \alpha^{\prime}=y_{\beta}$. Then $f[Z] \cap \alpha=y_{\beta} \cap \alpha$ and the conclusion follows immediately from the fact that $\alpha \in C_{f}$.

With the statement $\bigcirc_{\lambda}(S)$ in hand, one can reformulate the first step in Shelah's argument as the following proposition. It reduces the proof of $\nabla_{\lambda}(S)$ to the proof of $\bigcirc_{\lambda}(S)$ and works even for cardinals $\lambda$ that are not successors, which is slightly more than Shelah originally proved. The second step in Shelah's argument can then be viewed as a proof of $\bigcirc_{\kappa^{+}}(S)$ from the localized GCH. We will show how to obtain $\bigcirc_{\kappa^{+}}(S)$ from the additional assumption that $\square_{\kappa}^{*}$ holds in situations where the localized GCH does not seem to suffice.

Proposition 1.3. Let $\lambda$ be regular and $S \subseteq \lambda$ be stationary. Then

$$
\bigcirc_{\lambda}(S) \Longrightarrow \nabla_{\lambda}(S) \text {. }
$$

Proof. Let $\left\langle x_{\xi} \mid \xi<\lambda\right\rangle,\left\langle A_{\delta} \mid \delta \in S\right\rangle$ be a pair satisfying the conclusion of Lemma1.2. For $x \subseteq \lambda \times \lambda$ we write $(x)_{\xi}$ to denote $\{\zeta<\lambda \mid\langle\xi, \zeta\rangle \in x\}$. Consider sequences $\left\langle X_{\xi}, C_{\xi} \mid \xi<\theta\right\rangle$ of length $\theta \leq \lambda$ such that $X_{\xi} \subseteq \lambda, C_{\xi}$ is closed unbounded in $\lambda$ and, letting

$$
V_{\xi}^{\delta}=\left\{\langle\alpha, \beta\rangle \in A_{\delta} \times A_{\delta} \mid(\forall \eta<\xi)\left(X_{\eta} \cap \alpha=\left(x_{\beta}\right)_{\eta} \cap \alpha\right)\right\},
$$

for every $\xi<\theta$ and $\delta \in S \cap C_{\xi}$ either $\operatorname{dom}\left(V_{\xi+1}^{\delta}\right)$ is bounded in $\delta$ or else $V_{\xi}^{\delta} \supsetneqq V_{\xi+1}^{\delta}$. Notice that the non-strict inclusion $V_{\xi}^{\delta} \supseteq V_{\xi^{\prime}}^{\delta}$ holds anyway whenever $\xi \leq \xi^{\prime}$.

The crucial observation is that any sequence $\left\langle X_{\xi}, C_{\xi} \mid \xi<\theta\right\rangle$ as above has length strictly below $\lambda$. Assume for a contradiction that this fails, that is, there is such a sequence with $\theta=\lambda$. Let $S^{\prime}$ come from the application of Lemma 1.2 to the pair $\left\langle x_{\xi}\right\rangle_{\xi},\left\langle A_{\delta}\right\rangle_{\delta}$ and the set

$$
Z=\left\{\langle\xi, \zeta\rangle \mid \zeta \in X_{\xi}\right\}
$$


and let $\delta \in S^{\prime} \cap \triangle\left\{C_{\xi} \mid \xi<\lambda\right\}$ be such that $\delta>\kappa$ if $\lambda=\kappa^{+}$and $\delta$ is a cardinal if $\lambda$ is inaccessible. We have arbitrarily large $\alpha<\delta$ for which there exists $\beta<\delta$ such that $\alpha, \beta \in A_{\delta}$ and $Z \cap(\alpha \times \alpha)=x_{\beta} \cap(\alpha \times \alpha)$, so for each $\xi<\delta$ the set $\operatorname{dom}\left(V_{\xi}^{\delta}\right)$ is unbounded in $\delta$. Since $\delta \in S \cap C_{\xi}$ whenever $\xi<\delta$, from the properties of the sequence $\left\langle X_{\xi}, C_{\xi} \mid \xi<\theta\right\rangle$ we obtain $V_{\xi}^{\delta} \supsetneqq V_{\xi^{\prime}}^{\delta}$ whenever $\xi<\xi^{\prime}<\delta$. This is a contradiction, as $V_{\xi}^{\delta} \subseteq A_{\delta} \times A_{\delta}$ and $\operatorname{card}\left(A_{\delta}\right)<\operatorname{card}(\delta)$.

Pick a sequence $\left\langle X_{\xi}, C_{\xi} \mid \xi<\theta\right\rangle$ as in the previous paragraph which has no proper extension. Then $\theta<\lambda$. Letting

$$
D_{\delta}=\bigcup\left\{\left(x_{\beta}\right)_{\theta} \cap \alpha \mid\langle\alpha, \beta\rangle \in V_{\theta}^{\delta}\right\},
$$

the sequence $\left\langle D_{\delta} \mid \delta \in S\right\rangle$ is a $\nabla_{\lambda}(S)$-sequence. To see this, pick an arbitrary $X \subseteq \lambda$ and a closed unbounded $C \subseteq \lambda$. There exists some $\delta \in S \cap C$ such that $\operatorname{dom}\left(V_{\theta}^{\delta}\right)$ is unbounded in $\delta$ and $X \cap \alpha=\left(x_{\beta}\right)_{\theta} \cap \alpha$ for all $\langle\alpha, \beta\rangle \in V_{\theta}^{\delta}$, as otherwise we could extend the sequence $\left\langle X_{\xi}, C_{\xi} \mid \xi<\theta\right\rangle$ by letting $X_{\theta}=X$ and $C_{\theta}=C$, in contradiction with its maximality. But then $X \cap \delta=D_{\delta}$.

We now focus on proofs of $\bigcirc_{\lambda}(S)$. The point of introducing $\bigcirc_{\lambda}(S)$ is that it is often easier to give a direct proof of $\bigcirc_{\lambda}(S)$ than a direct proof of $\diamond_{\lambda}(S)$. This is clear from Shelah's argument in [10, which in our notation is a proof of $\bigcirc_{\kappa^{+}}(S)$. As Proposition 1.3 also holds for inaccessible $\lambda$, our hope was that Shelah's argument could be used for proofs of $\nabla_{\lambda}(S)$ for inaccessible $\lambda$. It seems, however, that for inaccessible $\lambda$ the proofs of $\nabla_{\lambda}(S)$ may require more new ideas. For instance, the proofs of $\nabla_{\lambda}\left(S_{\varepsilon}^{\lambda}\right)$ for a Mahlo cardinal $\lambda$ in [6] and 11 can be easily modified to give proofs of $\bigcirc_{\lambda}\left(S_{\varepsilon}^{\lambda}\right)$, but introducing a $\bigcirc_{\lambda}\left(S_{\varepsilon}^{\lambda}\right)$-sequence into the construction does not seem to enable any strengthening of the results or a simplification of the construction in [11. For inaccessible $\lambda$ that are not Mahlo it is not clear either whether an argument using $\bigcirc_{\lambda}\left(S_{\varepsilon}^{\lambda}\right)$ might work. It is certainly clear that constructions of a $\bigcirc_{\lambda}\left(S_{\varepsilon}^{\lambda}\right)$-sequence from "below" as in Propositions 1.4 and 1.5 will not work, essentially for the same reason why constructions of $\nabla_{\lambda}\left(S_{\varepsilon}^{\lambda}\right)$ from "below" cannot work, as described in [11. Analogously to 11], given any fixed $\bigcirc_{\lambda}\left(S_{\varepsilon}^{\lambda}\right)$-witness $\left\langle x_{\beta} \mid \beta<\lambda\right\rangle,\left\langle A_{\delta} \mid \delta<\lambda\right\rangle$, there is a $<\lambda$-distributive forcing that "kills" such a witness. On the other hand, any construction of a $\bigcirc_{\lambda}\left(S_{\varepsilon}^{\lambda}\right)$-witness from "below" would give rise to the same witness in the ground model and in the generic extension.

Let us turn to the proof of $\bigcirc_{\kappa^{+}}(S)$. As already mentioned above, the next proposition can be viewed as the first step in Shelah's argument. We include it, as it is a starting point for our variation using weak square.

Proposition 1.4. Assume $S \subseteq \kappa^{+}$is stationary and disjoint from $T_{\kappa}$. Then

$$
2^{\kappa}=\kappa^{+} \Longrightarrow \bigcirc_{\kappa^{+}}(S) \text {. }
$$

Proof. Pick an arbitrary enumeration $\left\langle y_{\xi} \mid \xi<\kappa^{+}\right\rangle$of $\left[\kappa^{+}\right] \leq \kappa$. The existence of such an enumeration is guaranteed by the localized GCH. Let $g: \varepsilon \times \kappa^{+} \rightarrow \kappa^{+}$be a bijection where $\varepsilon=\mathrm{cf}(\kappa)$. For each $\delta \in S$ pick an increasing (with respect to the inclusion) sequence of sets $\left\langle A_{\iota}^{\delta} \mid \iota<\varepsilon\right\rangle$ such that $\left|A_{\iota}^{\delta}\right|<\kappa$ for all $\iota<\varepsilon$ and $\bigcup_{\iota<\varepsilon} A_{\iota}^{\delta}=\delta$. 
We show that there is an $\iota<\varepsilon$ such that for every $Z \subseteq \kappa^{+}$there are stationarily many ordinals $\delta \in S$ satisfying:

For unboundedly many $\alpha<\delta$ there are $\beta<\delta$ such that

$$
\alpha, \beta \in A_{\iota}^{\delta} \text { and } Z \cap \alpha=\left(g^{-1}\left[y_{\beta}\right]\right)_{\iota} .
$$

It follows that upon letting $A_{\delta}=A_{\iota}^{\delta}$ and $x_{\beta}=\left(g^{-1}\left[y_{\beta}\right]\right)_{\iota}$, the pair $\left\langle x_{\beta} \mid \beta<\kappa^{+}\right\rangle$, $\left\langle A_{\iota}^{\delta} \mid \delta \in S\right\rangle$ witnesses $\bigcirc_{\kappa}+(S) !$

Assume for a contradiction that there is no $\iota$ as in the previous paragraph. Then for every $\iota<\varepsilon$ there is a set $Z_{\iota} \subseteq \kappa^{+}$such that (1.1) holds only on a non-stationary subset of $S$. Let $Z=\left\{\langle\iota, \xi\rangle \mid \xi \in Z_{\iota}\right\}$ and $Z^{\prime}=g[Z]$. The set $S^{\prime}$ consisting of all $\delta \in S$ such that

- $g[\varepsilon \times \alpha]=\alpha$ for cofinally many $\alpha<\delta$ and

- $(\forall \alpha<\delta)(\exists \beta<\delta)\left(Z^{\prime} \cap \alpha=y_{\beta}\right)$

is stationary in $\kappa^{+}$. For each $\delta \in S^{\prime}$ pick a cofinal strictly increasing sequence $\left\langle\alpha_{\eta}^{\delta} \mid \eta<\operatorname{cf}(\delta)\right\rangle$ such that $g\left[\varepsilon \times \alpha_{\eta}\right]=\alpha_{\eta}$ for each $\eta<\operatorname{cf}(\delta)$, and for each $\eta<\operatorname{cf}(\delta)$ pick $\beta_{\eta}<\delta$ such that $Z^{\prime} \cap \alpha_{\eta}=y_{\beta_{\eta}}$. This is possible by the above arrangements for elements of $S^{\prime}$.

If $\delta \in S^{\prime}$, then there is an $\iota(\delta)<\varepsilon$ such that $\alpha_{\eta}, \beta_{\eta} \in A_{\iota(\delta)}^{\delta}$ for cofinally many $\eta<\operatorname{cf}(\delta)$. This follows immediately if $\operatorname{cf}(\delta)<\varepsilon$, as the assignment

$$
\eta \mapsto \text { the least } \iota \text { such that } \alpha_{\eta}, \beta_{\eta} \in A_{\iota}^{\delta}
$$

cannot be cofinal in $\varepsilon$, so that in fact $\alpha_{\eta}, \beta_{\eta} \in A_{\iota(\delta)}^{\delta}$ for all $\eta<\operatorname{cf}(\delta)$. If $\operatorname{cf}(\delta)>\varepsilon$ this follows from the pigeonhole principle, namely that the inverse image of some $A_{\iota}^{\delta}$ under this assignment must have size $\operatorname{cf}(\delta)$. Applying the pigeonhole principle to the assignment $\delta \mapsto \iota(\delta)$, we obtain a stationary $S^{\prime \prime} \subseteq S^{\prime}$ and a $\iota<\varepsilon$ such that $\iota(\delta)=\iota$ for all $\delta \in S^{\prime \prime}$.

Pick $\delta \in S^{\prime \prime}$. By the above arrangements, there are cofinally many $\alpha<\delta$ for which there are $\beta<\delta$ such that $\alpha, \beta \in A_{\iota}^{\delta}$ and $Z^{\prime} \cap \alpha=y_{\beta}$. Moreover, the ordinals $\alpha$ can be chosen so that $g[\varepsilon \times \alpha]=\alpha$. It follows that

$$
Z \cap(\varepsilon \times \alpha)=g^{-1}\left[Z^{\prime} \cap \alpha\right]=g^{-1}\left[y_{\beta}\right],
$$

so $Z_{\iota} \cap \alpha=\left(g^{-1}\left[y_{\beta}\right]\right)$, for all $\alpha, \beta$ as above. Since this is true of any $\delta \in S^{\prime \prime}$ we have obtained a contradiction to the fact that $Z_{\iota}$ is a counterexample to (1.1).

The following proposition shows how to apply a standard construction that uses $\square_{\kappa}^{*}$ to prove $\bigcirc_{\kappa^{+}}(T)$.

Proposition 1.5. Assume $\kappa$ is singular and $T \subseteq T_{\kappa}$ is a stationary subset of $\kappa^{+}$ with stationarily many reflection points. Then

$$
2^{\kappa}=\kappa^{+}+\square_{\kappa}^{*} \Longrightarrow \bigcirc_{\kappa^{+}}(T) \text {. }
$$

Proof. We elaborate on the argument from the proof of Proposition 1.4, Let $\varepsilon=$ $\mathrm{cf}(\kappa)$. Fix the following objects:

- Sequences $\left\langle y_{\xi} \mid \xi<\kappa^{+}\right\rangle,\left\langle A_{\iota}^{\delta} \mid \iota<\varepsilon\right\rangle$ and a bijection $g: \varepsilon \times \kappa^{+} \rightarrow \kappa^{+}$as in the proof of Proposition 1.4.

- For each ordinal $\delta<\kappa^{+}$an injection $h_{\delta}: \delta \rightarrow \kappa$.

- $\mathrm{A} \square_{\kappa}^{*}$-sequence $\left\langle\mathcal{C}_{\delta} \mid \delta \in \lim \left(\kappa, \kappa^{+}\right)\right\rangle$. For each $\delta$ fix an enumeration $\left\langle c_{\zeta}^{\delta} \mid \zeta<\kappa\right\rangle$ of the set $\mathcal{C}_{\delta}$.

\footnotetext{
${ }^{1}$ See the proof of Proposition 1.3 for the notation $(u)_{\eta}$.
} 
- An increasing (with respect to the inclusion) sequence of sets $\left\langle B_{\iota} \mid \iota<\varepsilon\right\rangle$ such that $\left|B_{\iota}\right|<\kappa$ for each $\iota$ and $\bigcup_{\iota<\varepsilon} B_{\iota}=\kappa \times \kappa$.

For each $\delta \in \lim \left(\kappa, \kappa^{+}\right)$and $\zeta<\kappa$ define a function $f_{\zeta}^{\delta}: \delta \rightarrow \kappa \times \kappa$ and a sequence of sets $\left\langle A_{\zeta, \iota}^{\delta} \mid \iota<\varepsilon\right\rangle$ as follows:

$$
f_{\zeta}^{\delta}(\xi)=\left\langle\eta, h_{\gamma}(\xi)\right\rangle
$$

where $\gamma$ is the least element of $c_{\zeta}^{\delta}$ strictly above $\xi$ and $\eta=\operatorname{otp}\left(c_{\zeta}^{\delta} \cap \gamma\right)-1 \Omega$ and

$$
A_{\zeta, \iota}^{\delta}=\left(f_{\zeta}^{\delta}\right)^{-1}\left[B_{\iota}\right]
$$

Notice that each $f_{\zeta}^{\delta}$ is an injection. By our choice of the sets $B_{\iota}$ we then have $\left|A_{\zeta, L}^{\delta}\right|<\kappa$ and $\bigcup_{L<\varepsilon} A_{\zeta, L}^{\delta}=\delta$. We also have the following coherency property for the sets $A_{\zeta, L}^{\delta}$ : If $\bar{\delta}$ is a limit point of $c_{\zeta}^{\delta}$, then there is an ordinal $\bar{\zeta}<\kappa$ such that

$$
A_{\zeta, \iota}^{\delta} \cap \bar{\delta}=A_{\bar{\zeta},\llcorner}^{\bar{\delta}} .
$$

To see this notice first that if $\bar{\delta}$ is a limit point of $c_{\zeta}^{\delta}$, then there is $\bar{\zeta}<\kappa$ such that $c_{\zeta}^{\delta} \cap \bar{\delta}=c_{\bar{\zeta}}^{\bar{\delta}}$, and from the definition of $f_{\zeta}^{\delta}$ we immediately conclude that $f_{\bar{\zeta}}^{\bar{\delta}}=f_{\zeta}^{\delta} \uparrow \bar{\delta}$. The rest follows immediately from the definition of $A_{\zeta, \iota}^{\delta}$.

Fix an increasing sequence $\left\langle\kappa_{\iota} \mid \iota<\varepsilon\right\rangle$ that is cofinal in $\kappa$. For each $\delta \in T$ and $\iota<\varepsilon$ set $A_{\iota}^{\delta}=\bigcup_{\zeta<\kappa_{\iota}} A_{\zeta, \iota}^{\delta}$. Notice that $\left|A_{\iota}^{\delta}\right|<\kappa$, as $\left|A_{\zeta, \iota}^{\delta}\right| \leq\left|B_{\iota}\right|$ for all $\zeta<\kappa_{\iota}$. Following the ideas from the proof of Proposition 1.4 we prove that there is an $\iota<\varepsilon$ such that for every $Z \subseteq \kappa^{+}$there are stationarily many $\delta \in T$ satisfying:

For unboundedly many $\alpha<\delta$ there are $\beta<\delta$ such that

$$
\alpha, \beta \in A_{\iota}^{\delta} \text { and } Z \cap \alpha=\left(g^{-1}\left[y_{\beta}\right]\right)_{\iota} .
$$

It follows that upon letting $x_{\beta}=\left(g^{-1}\left[y_{\beta}\right]\right)_{\iota}$ and $A_{\delta}=A_{\iota}^{\delta}$ where $\iota$ is as above, the pair $\left\langle x_{\beta} \mid \beta<\kappa^{+}\right\rangle,\left\langle A_{\delta} \mid \delta \in T\right\rangle$ witnesses $\bigcirc_{\kappa^{+}}(T)$.

Assume for a contradiction that no $\iota$ as above exists. As in the proof of Proposition 1.4 pick a counterexample $Z_{\iota}$ for each $\iota<\varepsilon$, and let $Z=\left\{\langle\iota, \xi\rangle \in \varepsilon \times \kappa^{+} \mid \xi \in\right.$ $\left.Z_{\iota}\right\}$ and $Z^{\prime}=g[Z]$. Let $C$ be a closed unbounded subset of $\kappa^{+}$. By our assumption on $T$, there is a reflection point $\delta^{\prime}$ of $T$ such that:

- $\delta^{\prime}$ is a limit point of $C$.

- $g[\varepsilon \times \alpha]=\alpha$ for cofinally many $\alpha<\delta^{\prime}$.

- $\left(\forall \alpha<\delta^{\prime}\right)\left(\exists \beta<\delta^{\prime}\right)\left(Z^{\prime} \cap \alpha=y_{\beta}\right)$.

As $\delta^{\prime}$ is a reflection point of $T$, necessarily $\operatorname{cf}\left(\delta^{\prime}\right)>\varepsilon$. Pick an increasing sequence $\left\langle\alpha_{\eta} \mid \eta<\operatorname{cf}\left(\delta^{\prime}\right)\right\rangle$ cofinal in $\delta^{\prime}$ such that $g\left[\varepsilon \times \alpha_{\eta}\right]=\alpha_{\eta}$ for each $\eta<\operatorname{cf}\left(\delta^{\prime}\right)$. To each $\eta<\operatorname{cf}\left(\delta^{\prime}\right)$ assign some $\beta_{\eta}<\delta^{\prime}$ satisfying $Z^{\prime} \cap \alpha_{\eta}=x_{\beta_{\eta}}$. It is convenient to pick $\beta_{\eta}$ to be least possible. Since $\operatorname{cf}\left(\delta^{\prime}\right)>\varepsilon$, using the pigeonhole principle we conclude that there is some $\iota^{\prime}<\varepsilon$ such that $\alpha_{\eta}, \beta_{\eta} \in A_{0, \iota^{\prime}}^{\delta^{\prime}}$ for cofinally many $\eta<\operatorname{cf}\left(\delta^{\prime}\right)$. Let $\delta \in T \cap C \cap \lim \left(c_{0}^{\delta^{\prime}}\right)$ be a limit point of $\left\{\alpha_{\eta} \mid \alpha_{\eta}, \beta_{\eta} \in A_{0, \iota^{\prime}}^{\delta^{\prime}}\right\}$. Such a $\delta$ exists by our choice of $\delta^{\prime}$ and $\iota^{\prime}$ and by the fact that $T \cap \delta^{\prime}$ is stationary in $\delta^{\prime}$. Let $\xi<\kappa$ be such that $A_{\xi, \iota^{\prime}}^{\delta}=A_{0, \iota^{\prime}}^{\delta^{\prime}} \cap \delta$ and let $\iota(\delta)>\iota^{\prime}$ be such that $\kappa_{\iota(\delta)}>\xi$. The existence of such a $\xi$ follows from (1.2). Then $A_{0, \iota^{\prime}}^{\delta^{\prime}} \cap \delta \subseteq A_{\iota(\delta)}^{\delta}$, as $B_{\iota^{\prime}} \subseteq B_{\iota(\delta)}$.

The previous paragraph proves that there is a stationary $T^{\prime} \subseteq T$ such that for every $\delta \in T^{\prime}$ there is an $\iota(\delta)<\varepsilon$ such that for cofinally many $\alpha<\delta$ there are $\beta<\delta$ such that $\alpha, \beta \in A_{\iota(\delta)}^{\delta}, Z^{\prime} \cap \alpha=y_{\beta}$ and $g[\varepsilon \times \alpha]=\alpha$. The rest of the proof literally

${ }^{2}$ Notice that $\operatorname{otp}\left(c_{\zeta}^{\delta} \cap \gamma\right)$ is a successor ordinal if $\gamma$ is as above. 
follows the proof of Proposition 1.4 We first find a stationary $T^{\prime \prime} \subseteq T^{\prime}$ on which the $\iota(\delta)$ stabilize; let $\iota$ be the stabilized value. Then we unfold $Z^{\prime}$ and $y_{\beta}$ using $g$ and conclude that for $\alpha, \beta$ as above we have $Z_{\iota} \cap \alpha=\left(g^{-1}\left[y_{\beta}\right]\right)_{\iota}$. This yields a contradiction with the fact that $Z_{\iota}$ is a counterexample to (1.3).

\section{REFERENCES}

1. Cummings, J., Foreman, M. and Magidor, M., Squares, scales and stationary reflection, Journal of Mathematical Logic 1 (2001), 35-98. MR1838355 (2003a:03068)

2. Devlin, K. J., Constructibility, Springer, 1984. MR.750828 (85k:03001)

3. Devlin, K. J. and Johnsbräten, H., The Souslin Problem, Lecture Notes in Mathematics, 405, Springer, 1974. MR.0384542 (52:5416)

4. Gitik, M. and Rinot, A., The failure of diamond on a reflecting stationary set, to appear.

5. Gregory, J., Higher Souslin trees and the generalized continuum hypothesis, Journal of Symbolic Logic 41 (1976), 663-671. MR0485361 (58:5208)

6. Jensen, R., Diamond at Mahlo cardinals, handwritten notes, Oberwolfach, 1991.

7. Komjáth, P., Shelah's proof of diamond, unpublished.

8. Rinot, A., A relative to the approachability ideal, diamond and non-saturation, Journal of Symbolic Logic, to appear.

9. Shelah, S., On successors of singular cardinals, Logic Colloquium '78, Stud. Logic Foundations Math., 97, North-Holland, 1979, 357-380. MR567680 (82d:03079)

10. Shelah, S., Diamonds, Shelah Archive 922, http://shelah.logic.at.

11. Zeman, M., $\diamond$ at Mahlo cardinals, Journal of Symbolic Logic 65(4) (2000), 1813-1822. MR 1812181 (2002g:03107)

Department of Mathematics, University of California at Irvine, Irvine, California 92697

E-mail address: mzeman@math.uci.edu 